\title{
Zinostatin Stimalamer
}

National Cancer Institute

\section{Source}

National Cancer Institute. Zinostatin Stimalamer. NCI Thesaurus. Code C162544.

A highly lipophilic conjug ate protein comprised of the lipophilic antitumor protein, neocarzinostatin (NCS), conjug ated with a water-soluble copolymer of styrene-maleic acid (SMA) with potential antineoplastic activity. Upon intra-hepatic arterial administration, zinostatin stimalamer is deposited within tumor tissues, where the NCS moiety induces sequence-specific sing le and double-stranded breaks via free-radical based mechanisms, resulting in tumor cell death. 